\title{
Adaptive Mimo Controller Design for Chaos Synchronization in Coupled Josephson Junctions via Fuzzy Neural Networks
}

\author{
Tat-Bao-Thien NGUYEN* \\ Faculty of Electrical and Electronics Engineering, Ton Duc Thang University, Ho Chi Minh City, \\ Vietnam \\ *nguyentatbaothien@tdt.edu.vn \\ (Received: 16-February-2017; accepted: 29-April-2017; published: 8-June-2017) \\ DOI: http://dx.doi.org/10.25073/jaec.201711.52
}

\begin{abstract}
In this paper, we have discussed the synchronization between coupled Josephson Junctions which experience different chaotic oscillations. Due to potential high-frequency applications, the shunted nonlinear resistivecapacitive-inductance junction (RCLSJ) model of Josephson junction was considered in this paper. In order to obtain the synchronization, an adaptive MIMO controller is developed to drive the states of the slave chaotic junction to follow the states of the master chaotic junction. The developed controller has two parts: the fuzzy neural controller and the sliding mode controller. The fuzzy neural controller employs a fuzzy neural network to simulate the behavior of the ideal feedback linearization controller, while the sliding mode controller is used to ensure the robustness of the controlled system and reduce the undesired effects of the estimate errors. In addition, the Lyapunov candidate function is also given for further stability analysis. The numerical simulations are carried out to verify the validity of the proposed control approach.
\end{abstract}

\section{Keywords}

Chaos Synchronization; Chaotic Systems; Fuzzy Neural Networks; Josephson Junction.

\section{Introduction}

Since Josephson Junction (JJ) possesses the advanced characteristics such as ultra-low noise, low power consumption and high working frequency [1], JJ has received much attention from many researchers. Then different models have been introduced to represent JJ [1, 2, [3, 4], 5]. Among many types of JJ models, two types of JJ models have attracted more researchers due to their exact modeling in JJ behaviors. These models are the shunted linear resistivecapacitive junction (RCSJ) and the shunted nonlinear resistive- capacitive-inductance junction (RCLSJ). The RCSJ model is the second order system while the RCLSJ model is the third order system. The RCLSJ model is found to be more accurate in high frequency applications [3], [4. Because the RCLSJ model is the third order system, this model can exhibit chaos even with external dc current only. The chaotic behavior of the RCLSJ model has been extensively studied by Dana, et al. 5. Afterward, there have been some control methods developed to control or synchronize RCLSJ model of Josephson Junction such as nonlinear feedback control [6], backstepping control [7, 8], delay linear feedback control [9, time delay feedback control [10] and sliding mode control 11]; however, some shortcomings exist. The nonlinear backstepping method has quite complicated procedure to design the controller while choosing the time de- 
lay is problematic in delay linear feedback. The chattering phenomenon is a drawback of the sliding mode method. Moreover, these control techniques almost require the exact mathematical models to design the controllers. This requirement becomes the significant limitation in design a nonlinear controller when the system parameters are unknown or the system is effected by uncertainties.

Nowadays, fuzzy logic and neural networks are used as the power tools for modelling and controlling highly uncertain, nonlinear and complex systems [12], [13], 14], [15], [16]. In this study, the chaos synchronization of coupled RCLSJ modes is expected. The synchronization can be obtained when the slave follows the master as close as possible. Based on fuzzy neural networks, we develop a MIMO controller that can force the states of slave to track the states of master with zero convergence of state errors. In this manner the chaos synchronization is obtained.

The remainder of this paper is organized as follows. In Section 2, the mathematical model of RCLSJ is described. The MIMO fuzzy neural controller design is presented in Section 3 with the numerical simulations are given in Section 4 . Finally, the conclusion is given Section 5 .

\section{The RCLSJ Model of Josephson Junction}

In high frequency application, the RCLSJ model of Josephson Junction is found more accuracy and appropriate than others [3, 4]. In the dimensionless form, the mathematical model of RCLSJ is given as follows [5]:

$$
\begin{aligned}
& z_{1}=z_{2}, \\
& z_{2}=\frac{1}{\beta_{C}}\left[i_{z}-g\left(z_{2}\right) z_{2}-\sin \left(z_{1}-z_{3}\right)\right], \\
& z_{3}=\frac{1}{\beta_{L}}\left[z_{2}-z_{3}\right],
\end{aligned}
$$

where state variables $z_{1}, z_{2}$ and $z_{3}$ represent the phase difference, junction voltage and current through shunted inductance, respectively.
$\beta_{C}$ and $\beta_{L}$ correspond to capacitive and inductance constants respectively and are considered as model parameters. $i_{z}$ stands for the external current consisting of a dc component only. The nonlinear damping term $g\left(z_{2}\right)$ is approximated with current voltage relation between the two junction resistances and is described by the following step function:

$$
g\left(z_{2}\right)= \begin{cases}0.366 & \text { if } z_{2}>2.9 \\ 0.061 & \text { if } z_{2} \leq 2.9\end{cases}
$$

The dynamics of RCLSJ model was extensively studied in [5]. This study demonstrated that the RCLSJ model can produce chaotic oscillations when the external dc current and the parameters fall into a certain area. For examples, the junction in Eq. (1) with zero initial states exhibits chaos when $\beta_{C}=0.707, \beta_{L}=2.6$, $i_{z}=1.2$ and as shown in Fig. 1 .

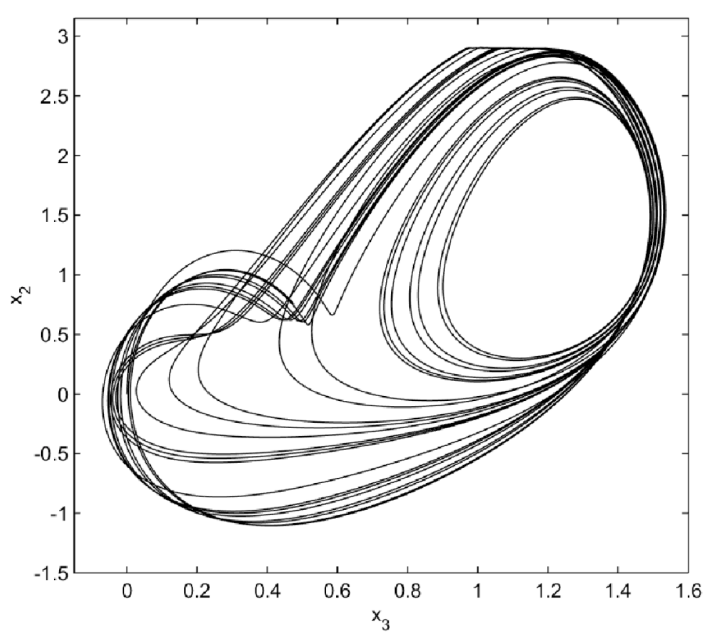

Fig. 1: Chaotic motion in Josephson Junction.

Remark 1. The dynamics of JJ much depends on their circuit parameters, including $\beta_{L}$ and $\beta_{C}$, and the external DC current $i_{z}$. The JJ shows the chaotic behaviors when these parameters fall into the chaotic region. This chaotic region can be referred in Figs. 9-10 of [4]. 


\section{Synchronization of the Coupled RCLSJ Models}

\subsection{Problem statement and preliminaries}

Consider the RCLSJ chaotic system defined in Eq. (1) as the master system with which the slave system need to be synchronized.

Consider the second RCLSJ chaotic system that contains the different values of initial conditions and external current as follows:

$$
\begin{aligned}
& x_{1}=x_{2}+u_{1}, \\
& x_{2}=\frac{1}{\beta_{C}}\left[i_{x}-g\left(x_{2}\right) x_{2}-\sin \left(x_{1}\right)-x_{3}\right]+u_{2}, \\
& x_{3}=\frac{1}{\beta_{L}}\left[x_{2}-x_{3}\right]+u_{3},
\end{aligned}
$$

where $u_{1}, u_{2}$ and $u_{3}$ are control signals. Here, the aim of these control signals is to force the state variables of the slave system described by Eq. (2) to follow the state variables of the master system given by Eq. (1) as close as possible. Thus, one-way synchronization of the two RCLSJ chaotic systems will be achieved. Since all state variables of the slave system are considered as outputs, the slave system with control inputs can be rewritten in the MIMO form as:

$$
\left\{\begin{array}{l}
\mathbf{x}=\mathbf{f}(\mathbf{x})+\mathbf{g}(\mathbf{x}) \mathbf{u} \\
\mathbf{y}=\mathbf{h}(\mathbf{x})
\end{array}\right.
$$

where

$$
\begin{aligned}
& \mathbf{x}=\left[\begin{array}{lll}
x_{1} & x_{2} & x_{3}
\end{array}\right]^{T}, \mathbf{y}=\left[\begin{array}{lll}
y_{1} & y_{2} & y_{3}
\end{array}\right]^{T}, \\
& \mathbf{h}(\mathbf{x})=\left[\begin{array}{lll}
x_{1} & x_{2} & x_{3}
\end{array}\right]^{T}, \mathbf{u}=\left[\begin{array}{lll}
u_{1} & u_{2} & u_{3}
\end{array}\right]^{T}, \\
& \mathbf{f}(\mathbf{x})=\left[\begin{array}{c}
x_{2} \\
1 / \beta_{C}\left[\begin{array}{c}
i_{x}-g\left(x_{2}\right) x_{2}-\sin \left(x_{1}\right)-x_{3} \\
1 / \beta_{L}\left[x_{2}-x_{3}\right.
\end{array}\right]
\end{array}\right], \\
& \mathbf{g}(\mathbf{x})=\left[\begin{array}{lll}
1 & 0 & 0 \\
0 & 1 & 0 \\
0 & 0 & 1
\end{array}\right] .
\end{aligned}
$$

Due to the relative degree of the system given by Eq. (3) $r_{1}=r_{2}=r_{3}=1$, the outputs of the slave system can be rewritten as:

$$
\mathbf{y}=\mathbf{f}(\mathbf{x})+\mathbf{g}(\mathbf{x}) \mathbf{u}
$$

Now, we define the errors between the dependent variables of master and slave as:

$$
\mathbf{e}=\mathbf{y}-\mathbf{y}_{\mathbf{d}} .
$$

where $\mathbf{e}=\left[\begin{array}{lll}e_{1} & e_{2} & e_{3}\end{array}\right]^{T}$ and $\mathbf{y}_{\mathbf{d}}=\left[\begin{array}{lll}z_{1} & z_{2} & z_{3}\end{array}\right]^{T}$.

Then, in order to meet the control objective, we use the input-output linearization technique and the nonlinear feedback controller can be obtained as [17]:

$$
\mathbf{u}^{*}=\mathbf{g}^{-\mathbf{1}}(\mathbf{x})[-\mathbf{f}(\mathbf{x})+\mathbf{v}(\mathbf{t})]
$$

where $\mathbf{v}(\mathbf{t})$ is the new input variable and it is given as:

$$
\mathbf{v}(\mathbf{t})=\mathbf{y}_{\mathbf{d}}-\mathbf{k e}
$$

where $\mathbf{k}=\operatorname{diag}\left(k_{1}, k_{2}, k_{3}\right)$ is positive defined matrix.

Substituting Eq. (6) into Eq. (4), we can get:

$$
\mathbf{y}=\mathbf{v}(\mathbf{t})
$$

Substituting Eq. (7) into Eq. (8), and using Eq. (5) implies that:

$$
\mathbf{e}+\mathbf{k e}=\mathbf{0} .
$$

The equation in Eq. (9) implies that $e_{j}$ with $j=1,2,3$ converges to zero exponentially. However, the ideal nonlinear controller in Eq. (6) can no longer be used when $\mathbf{f}(\mathbf{x})$ and $\mathbf{g}(\mathbf{x})$ in Eq. (3) change their values and become unknown due to parameter perturbation and noise disturbance. In order to bypass this control problem, a fuzzy neural network was used to directly approximate the values of control signals. 


\subsection{Designed fuzzy neural network}

Since fuzzy logic and neural networks have exhibited the superior abilities in modeling and controlling the highly uncertain, ill-defined and complex systems, we employ a fuzzy neural network which combines the advantageous merits of a fuzzy logic system and a neural network to approximate the nonlinear control laws $u_{1}, u_{2}$ and $u_{3}$. The structure of the fuzzy neural network is depicted in Fig. 2

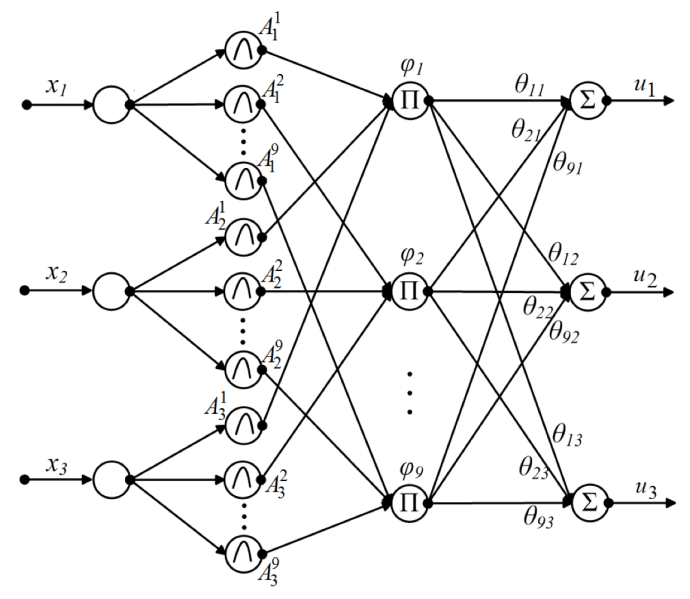

Input layer Membership layer Rule layer Output layer

Fig. 2: Structure of the designed fuzzy neural network.

This network structure has four layers: input layer, membership layer, rule layer and output layer. Nodes in the input layer are 3 state variables of the slave chaotic JJ and their values are directly transmitted to the membership layer. When 9 fuzzy rules were used for network design, the membership layer has $3 \times 9$ nodes. Each node performs a membership function and employs a Gaussian function to calculate its value. The rule layer has 9 nodes and each node corresponds to an element $\psi(\mathbf{x})$ of the fuzzy basis vector $\psi(\mathbf{x})$ and performs a fuzzy rule. Thus, in the rule layer, all nodes denote the fuzzy rule set. The output layer is connected to the rule layer through weighting factors, $\theta_{i j}$ with $i=19, j=1$ 3 . The weighting factors $\theta_{i j}$ are elements of the weighting vector $\theta(t)$. These factors are the parameters of the networks and they will be tuned by designed adaptive laws given in Eq. (11). In the output layer, 3 nodes act for the values of control signals $u_{1}, u_{2}$ and $u_{3}$ at time t.

\subsection{Adaptive fuzzy controller design}

When $f(x)$ and $g(x)$ are unknown, the ideal control law in Eq. (6) cannot be determined, and therefore this control law cannot be used. To solve this problem, we developed a fuzzy neural network to directly approximate the nonlinear control law. In order to ensure our design proper, we need the following assumptions.

Assumption 1. The scalar matrix $\mathbf{g}(\mathbf{x})$ is positive defined, then it exists some positive constants $\underline{g}, \bar{g} \in R$ such that $\underline{\mathbf{g}} \mathbf{I} \leq \mathbf{g}(\mathbf{x}) \leq \overline{\mathbf{g} I}$.

Assumption 2. The rate of variation of $\mathbf{g}(\mathbf{x})$ is bounded, that is, there exists a constant $D \in$ $R$ such that $|g(x)| \leq D \mathbf{I}$.

Let the fuzzy neural controller $\mathbf{u}_{\mathbf{f}}$ be the approximation of the ideal controller given in Eq. (6). $\mathbf{u}_{\mathbf{f}}$ is online estimated by a fuzzy neural network as follows:

$$
\mathbf{u}_{\mathbf{f}}=\theta^{\mathbf{T}}(\mathbf{t}) \psi(\mathbf{x})
$$

where $\left[\begin{array}{ccc}\theta_{11} & \ldots & \theta_{13} \\ \vdots & & \vdots \\ \theta_{91} & \ldots & \theta_{93}\end{array}\right]$ is weighting matrix of which each entry is represented by a link between Rule layer and Output layer in the chosen fuzzy neural network. $\psi(\mathbf{x})=\left[\begin{array}{ll}\psi_{1} & \psi_{1}\end{array}\right]^{T}$ is fuzzy basic vector of which each element $\psi_{i}$ with $i=1$ 9 is defined as:

$$
\varphi_{i}(x)=\frac{\prod_{j=1}^{3} \mu_{A_{j}^{i}}(x)}{\sum_{i=1}^{9}\left(\prod_{j=1}^{3} \mu_{A_{j}^{i}}(x)\right)}
$$

where the membership functions $\mu_{A_{j}^{i}}(x)$ 's employ Gaussian function to calculate their values.

The adaptive law which allows the weighting matrix $\theta(\mathbf{t})$ to vary so that the fuzzy neural controller $\mathbf{u}_{\mathbf{f}}$ reaches the ideal controller $\mathbf{u}^{*}$ is chosen as: 


$$
\dot{\theta}_{i j}=-w_{i j} \psi_{i} e_{j} \quad \text { with } i=1 \quad 9, j=13
$$

where $w_{i j}$ s are positive factors which govern the rate of adaption.

Since the designed fuzzy neural network has the finite number of units in the hidden layer, the approximation errors are unavoidable. We assume that, these approximation errors are bounded by a known vector $\bar{\delta}=\left[\begin{array}{lll}\overline{\delta_{1}} & \overline{\delta_{2}} & \overline{\delta_{3}}\end{array}\right]$. Then a sliding mode controller $\mathbf{u}_{\mathbf{s}}$ is added to reduce the undesirable effects of the approximation errors. The formula of $\mathbf{u}_{\mathbf{s}}$ is given as:

$$
u_{s}=-\operatorname{diag}(\operatorname{sgn}(e))\left(\bar{\delta}+\frac{D}{2 \underline{g}^{2}}|e|\right)
$$

where $|$.$| denotes the absolute value.$

From Eq. (10) and Eq. (12), the total controller is achieved as:

$$
\begin{gathered}
u=u_{f}+u_{s} \\
=\theta^{T}(t) \psi(x)-\operatorname{diag}(\operatorname{sgn}(e))\left(\bar{\delta}+\frac{D}{2 \underline{g}^{2}}|e|\right) .
\end{gathered}
$$

Therefore, the coupled RCLSJ models can be synchronized with the control law in Eq. (13) and the adaptive mechanism in Eq. (11).

Moreover, for stability analysis, the Lyapunov approach can be used. First, the Lyapunov candidate function can be considered as follows:

$$
V=\frac{1}{2} \mathbf{e}^{T} \mathbf{g}^{-1} \mathbf{e}+\frac{1}{2} \sum_{i=1}^{9} \sum_{j=1}^{3} \frac{1}{w_{i j}} \tilde{\theta}_{i j}^{T} \theta_{i j},
$$

where $\tilde{\theta}_{i j}$ is a parameter error between the current paramter $\theta_{i j}$ and optimal papameter $\theta_{i j}^{*}$. Notice that the optimal papameter $\theta_{i j}^{*}$ is an artificial constant quantity introduced only for analytical purpose and it is not needed for implemantation. Taking some algebraic manipulations and incorporating the control law in Eq. 13 and the adaptive law in Eq. (11), one can get the time derivative which is less than zero as:

$$
V=-\frac{e^{T} k e}{\bar{g}} \leq 0 .
$$

Since the chosen Lyapunov candidate function is positive and its time derivative is less than zero, the controlled system is stable.

\section{Numerical Simulations}

In this section, the numerical results are given to verify the proposed method. In order to demonstrate the procedure, we keep the zero initial conditions and the external current $i_{z}=1.2$ for the master system. Then we choose the different values for the slave system, that is $\left[\begin{array}{lll}x_{1} & {\left[x_{2}\right.} & {\left[x_{3}\right.}\end{array}\right]^{T}=\left[\begin{array}{lll}1 & 1 & 1\end{array}\right]^{T}$ and $i_{x}=1.135$. The model parameters $\beta_{L}=2.6$ and $\beta_{C}=0.707$ are chosen and fixed for both master and slave. Because of the different values of external dc currents and initial conditions, the master and slave produce chaotic oscillations differently.

First, the coupled systems are considered in the case of without control signals. Due to the different chaotic motions between master and slave, the chaotic oscillations are reflected into state errors as shown in Fig. 3
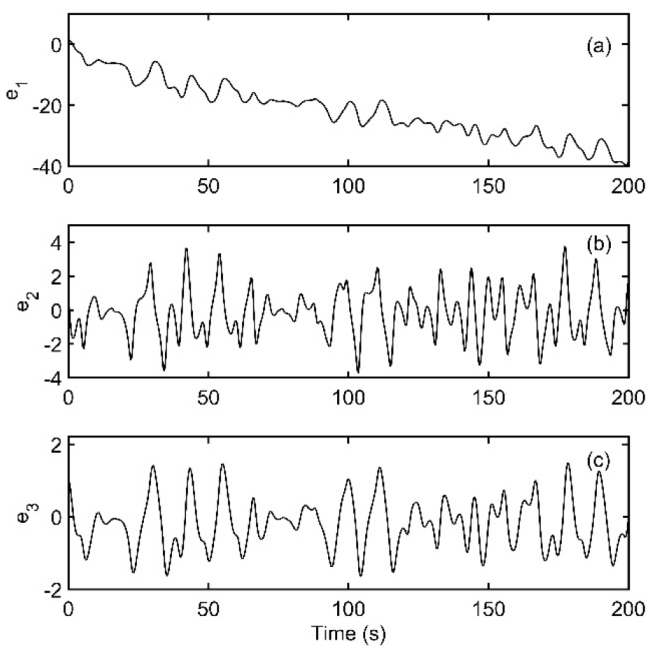

Fig. 3: The state errors between master and slave without control effects.

Second, the coupled systems are controlled by the MIMO fuzzy neural controller. In this 
case, some controller parameters are chosen as: $\mathrm{w}_{\mathrm{ij}}=5000, \mathrm{D}=3, \bar{\delta}_{\mathrm{j}}=0.05$ and $\mathrm{g}=0.5$. Under the effects of the controller, the state errors between the master and the slave, as depicted in Fig. 4, converge to zero quickly. By this way, the chaos synchronization of coupled RCLSJ models is obtained.

Finally, we assume that the slave JJ is influenced by noises at its inputs. In this case, the white noise with zero mean and variance $\sigma=0.5$ is chosen for simulation. The controller parameter are maintained as given above. The simulation results, as shown in Fig. 5 express that the state errors can well converge to zero even with the effects of input noises. This illustrates the robustness of the proposed controller.

Remark 2. The factors $\mathrm{w}_{\mathrm{ij}}$ influence the rate of adaptation according to Eq. (11). When the factors $\mathrm{w}_{\mathrm{ij}}$ are large, the tracking errors can converge to fast. In this situation, the controlled systems are very sensitive to noises. The parameter is related to the signals of the sliding mode controller as given in Eq. (12). The large $D$ leads to the large control signal produced by the sliding mode controller. $\delta_{j, j}=1-3$ are upper bounds of approximation errors of the network. The values of $\delta_{j, j}=1-3$ are arranged such that they are close to the real errors of the designed network. The parameter $\mathrm{g}$ is lower bound of the unknown function $\mathbf{g}(\mathbf{x})$. The value of $\underline{g}$ can be chosen according to the experiences of technicians.

\section{Conclusion}

In this paper, we successfully developed the MIMO fuzzy neural controller to synchronize two RCLSJ chaotic systems together. The advanced abilities of the developed MIMO controller allows the slave chaotic system to follow the master chaotic system very fast. In contrast to the results in [6], our designs allow the coupled chaotic JJ to synchronize even when the models of JJ are unknown or are under the influence of the parameter perturbations. Finally, the simulation results illustrated the advanced abilities of the developed controller.
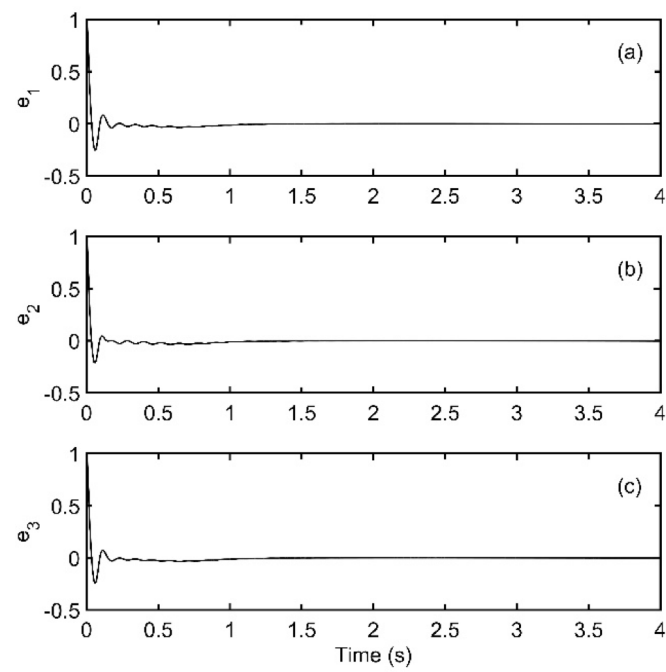

Fig. 4: The state errors between master and slave under the effects of controller.
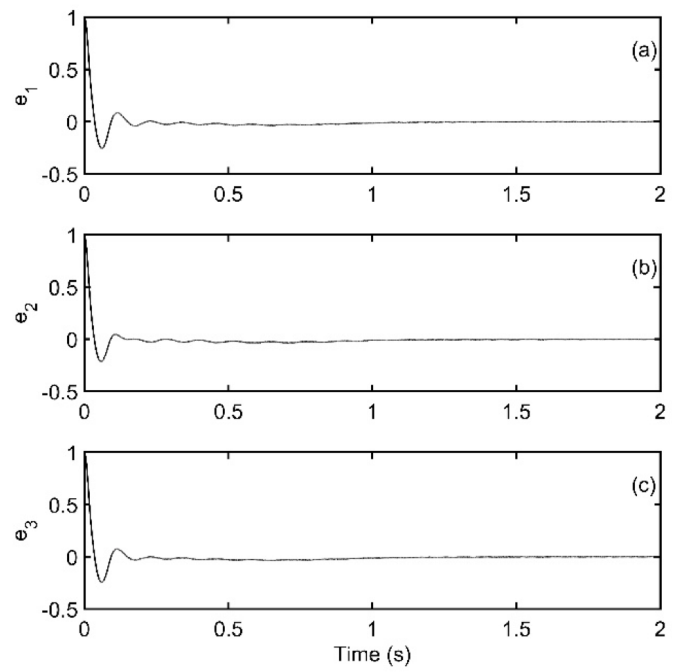

Fig. 5: The state errors between master and slave under the effects of controller in the case of input noises.

\section{References}

[1] LIKHAREV, K. K. Dynamics of Josephson junction and circuits, Gordon and Breach, 1986.

[2] CIRILLO, M. and N. F. PEDERSEN. "On bifurcations and transition to chaos in a Josephson junction," Physics Letters A, vol. 
90, pp. 150-152, 6/28/ 1982 .

[3] WHAN, C. B. and C. J. LOBB. "Complex dynamical behavior in RCL-shunted Josephson junctions," Applied Superconductivity, IEEE Transactions on, vol. 5, pp. 3094-3097, 1995.

[4] CAWTHORNE, A. B., C. B. WHAN and C. J. LOBB, "Complex dynamics of resistively and inductively shunted Josephson junctions," Journal of Applied Physics, vol. 84, pp. 1126-1132, 1998.

[5] DANA, S. K., D. C. SENGUPTA and K. D. EDOH. "Chaotic dynamics in Josephson junction," Circuits and Systems I: Fundamental Theory and Applications, IEEE Transactions on, vol. 48, pp. 990-996, 2001.

[6] A. UCAR, K. E. LONNGREN and E.W. BAI, "Chaos synchronization in RCLshunted Josephson junction via active control," Chaos, Solitons \& Fractals, vol. 31, pp. 105-111, 1/2007.

[7] HARB, A. M. and B. A. HARB. "Controlling Chaos in Josephson-Junction Using Nonlinear Backstepping Controller," $A p$ plied Superconductivity, IEEE Transactions on, vol. 16, pp. 1988-1998, 2006.

[8] HARB, A. and B. HARB, "Chaos Synchronization in Josephson Junctions," Journal of Superconductivity and Novel Magnetism, vol. 25, pp. 1647-1653, 2012.

[9] FENG, Y. L. and K. SHEN. "Controlling chaos in RCL-shunted Josephson junction by delayed linear feedback," Chinese Physics B, vol. 17, 2008.

[10] XU, S., Y. TANG, H. SUN, Z. ZHOU and Y. YANG. "Characterizing the anticipating chaotic synchronization of RCL-shunted Josephson junctions," International Journal of Non-Linear Mechanics, vol. 47, pp. 1124-1131, 2012.

[11] CHEN, D.-Y., W.-L. ZHAO, X.-Y. MA and R.-F. ZHANG. "Control and Synchronization of Chaos in RCL-Shunted Josephson Junction with Noise Disturbance Using Only One Controller Term," Abstract and Applied Analysis, vol. 2012, p. 14, 2012.
[12] WANG, L. X. A Course in Fuzzy Systems and Control, Prentice Hall PTR, 1997.

[13] NGUYEN, T.-B.-T., T.-L. LIAO and J.J. YAN. "Adaptive tracking control for an uncertain chaotic permanent magnet synchronous motor based on fuzzy neural networks," Journal of Vibration and Control, July 8, 2013.

[14] NGUYEN, T.-B.-T., T.-L. LIAO, H.-H. KUO, and J.-J. YAN. "An Improved Adaptive Tracking Controller of Permanent Magnet Synchronous Motor," Abstract and Applied Analysis, vol. 2014, p. 12, 2014.

[15] NGUYEN, T.-B.-T., T.-L. LIAO and J.-J. YAN. "Adaptive Sliding Mode Control of Chaos in Permanent Magnet Synchronous Motor via Fuzzy Neural Networks," Mathematical Problems in Engineering, vol. 2014, p. 11, 2014.

[16] NGUYEN, T.-B.-T., T.-L. LIAO and J.J. YAN. "Improved Adaptive Sliding Mode Control for a Class of Uncertain Nonlinear Systems Subjected to Input Nonlinearity via Fuzzy Neural Networks," Mathematical Problems in Engineering, vol. 2015, p. 13, 2015.

[17] SLOTINE, J. J. E. and W. LI. Applied Nonlinear Control, Pearson Education Taiwan Ltd, 2005.

\section{About Authors}

Tat-Bao-Thien NGUYEN received a Ph.D. degree in Electrical Control and Communication from National Cheng Kung University, Taiwan, on December 2014. Since 2016, he has been a lecturer at the Department of Electrical and Electronics Engineering, Ton Duc Thang University, Vietnam. His current research interests include automatic control, embedded systems and robotics.

"This is an Open Access article distributed under the terms of the Creative Commons Attribution License, which permits unrestricted use, distribution, and reproduction in any medium, provided the original work is properly cited (CC BY 4.0)." 